\title{
Fatigue Performances of Steel Box Girder in Orthotropic Steel Bridge Deck with Corrosive Environment
}

\author{
Lin Su, a, Jian Zhang ${ }^{2, b}$, Wanghua Yin ${ }^{2, c}$ and Lei Jiang ${ }^{2, d}$ \\ 1Jiangsu Yangtze River Bridge Co., Ltd., Jiangsu Nanjing, China \\ ${ }^{2}$ Nanjing University of Aeronautics and Astronautics, Jiangsu Nanjing, China \\ asulinjydq@126.com, bjianzhang78@126.com, cyinwh@163.com, djianglnuaa@163.com
}

\begin{abstract}
Keywords: Fatigue, Corrosive Environment, Steel Box Girder
Abstract. According to available results of the engineering investigation, the fatigue cracks are inevitably existed in steel bridges because of the environmental pollution, overloading vehicles and larger numbers of automobiles. The static and fatigue tests of the orthotropic steel bridge deck models were carried out in this research with the fatigue damage developing laws. An experiment design of corrosive and fatigue property of steel box girder with the specific environment controlling equipment is herein taken into account. Through the data from the testing design, the important characteristic width and length of the fatigue crack can be achieved, which can be used for the grasping of the mechanism of the steel box girder in the future.
\end{abstract}

\section{Introduction}

The steel bridge structure should not only inherit the crowd, vehicle load etc, but also inherit chemical corrosion such as the Marine environment, atmospheric environment, ice, salt. Europe, Japan and other countries began studying the fatigue of steel box girder in steel bridge, they just find a few parts of the steel box girder is easy to damage without considering the external environment in the process of research on the influence of the steel box girder[1-2]. Chinese scholars also began to research various structural details of steel box girder, but they are also no considering the external environment on the influence of the steel box girder. It is summarized that the cause of fatigue crack of orthotropic steel bridge panel is that the principal stress and surface deformation [3-4]. In china academy of railway sciences the mechanical characteristics of the orthotropic steel bridge panel by the static experiment of the real bridge, dynamic experiment of the real bridge, and the full-scale model static test were all studied [5-6]. The stress status of the field experiment and theoretical analysis to an across 40 meters box girder bridge of steel bridge deck in under the action of a three axis of heavy truck were also researched which was shown that the fatigue calculation of steel bridge panel don't consider more effect. Due to the superior structure style, orthotropic steel bridge deck has been getting more and more widely practical application. Because of the complex load distribution, multivariable structural form, stress concentration in welding site and inevitable welding flaws, fatigue failure tends to happen in bridge decks. Steel box girder generally is composed of roof, floor, web stiffeners, horizontal clapboard, web plate and stiffener[7-8]. The use of steel box girder bridges as the main load-bearing components can be traced back to Germany after the second world war, because of the shortage of steel, German needs to build and rebuild a large number of bridges to restore the post-war economy. In order to solve this problem, Germany began using a new type of bridge bearing structure which is designed by its high strength steel and welding technology, and the new structure is called as steel box girder. Then the engineers from all over the world gradually found the advantages of steel box girder, steel box girder widely used in domestic bridge construction. Up to the present, No fatigue design standard on orthotropic steel bridge deck has appeared in any country's bridge design code. Further research needs to be carried out both on stress amplitude definition and detail constitution classification.

In the research domains such as aeronautics and astronautics, steel structures are widely existed. Factually, fatigue testing system has been developed and applied successfully. But in steel box girder of bridge engineering, there is no result about fatigue testing system emerging. the static and fatigue 
tests of the orthotropic steel bridge deck models were carried out in this research paper, which presented the fatigue damage developing laws. And the corrosion experiment design is worked out.

\section{Analysis of fatigue strain}

In elastic load range, the fatigue strain is achieved through the fatigue test on orthotropic steel bridge deck. Due to the material mechanics, three linear strains called $\varepsilon_{\alpha 1}, \varepsilon_{\alpha 2}$ and $\varepsilon_{\alpha 3}$ are along the three directions $\alpha_{1}, \alpha_{2}$ and $\alpha_{3}$ of a selected point. The relationships between these variables $\varepsilon_{\mathrm{x}}, \varepsilon_{\mathrm{y}}$ and $\varepsilon_{\mathrm{z}}$ are as follows:

$$
\begin{aligned}
& \left.\begin{array}{l}
\varepsilon_{\text {max }} \\
\varepsilon_{\text {min }}
\end{array}\right\}=\frac{\varepsilon_{\mathrm{x}}+\varepsilon_{\mathrm{y}}}{2} \pm \sqrt{\left(\frac{\varepsilon_{\mathrm{x}}-\varepsilon_{\mathrm{y}}}{2}\right)^{2}+\left(\frac{\gamma_{\mathrm{xy}}}{2}\right)^{2}} \\
& \tan 2 \alpha_{0}=-\frac{\gamma_{\mathrm{xy}}}{\varepsilon_{\mathrm{x}}-\varepsilon_{\mathrm{y}}}
\end{aligned}
$$

Due to $\alpha_{1}=0^{\circ}, \alpha_{2}=45^{\circ}$ and $\alpha_{3}=90^{\circ}$, we have:

$$
\begin{aligned}
& \left.\begin{array}{l}
\varepsilon_{\max } \\
\varepsilon_{\min }
\end{array}\right\}=\frac{\varepsilon_{0^{\circ}}+\varepsilon_{90^{\circ}}}{2} \pm \frac{\sqrt{2}}{2} \sqrt{\left(\varepsilon_{0^{\circ}}-\varepsilon_{45^{\circ}}\right)^{2}+\left(\varepsilon_{45^{\circ}}-\varepsilon_{90^{\circ}}\right)^{2}} \\
& \tan 2 \alpha_{0}=\frac{2 \varepsilon_{45^{\circ}}-\varepsilon_{0^{\circ}}-\varepsilon_{90^{\circ}}}{\varepsilon_{0^{\circ}}-\varepsilon_{90^{\circ}}}
\end{aligned}
$$

The Von.Mises is gained as:

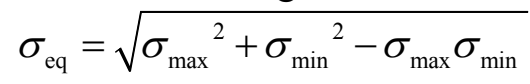

\section{Specimen Design}

The sectional steel box girder specimen is designed by referring to the sectional steel box girder of Jiangyin Yangtze bridge, and its shape and size as shown in Fig. 1. The specimen is welded by the roof and U longitudinal, the materials of the specimens is Q345b steel, the welding material is E50.

The coating solutions of specimen is as follows: The external of $U$ longitudinal is sprayed 3 layers, zinc silicate shop primer is $15 \mu \mathrm{m}$, epoxy iron oxide intermediate paint is $150 \mu \mathrm{m} \mathrm{s}$, epoxy is $100 \mu \mathrm{m}$; The interior of $U$ longitudinal is sprayed llayers, zinc silicate shop primer is $15 \mu \mathrm{m}$. Steel box girder bridge deck driveway is sprayed 3 layers, zinc silicate shop primer is $15 \mu \mathrm{m}$, , epoxy zinc rich primer is $75 \mu \mathrm{m}$, high solids high build epoxy tar paint is $250 \mu \mathrm{m}$.

This experiment is mainly experiment the fatigue strength about the groove welding between the longitudinal ribs and bridge panel. So the longitudinal ribs and the panel are strain gages, and the position of the strain gauge paste take away from the groove weld root $15 \mathrm{~mm}$. Along the longitudinal direction, four groups of strain gages along the longitudinal strain gauge for $30 \mathrm{~mm}, 90 \mathrm{~mm}, 150 \mathrm{~mm}$ and $210 \mathrm{~mm}$ is pasted. Each specimen has 24 strain gages, as shown in Fig. 2.
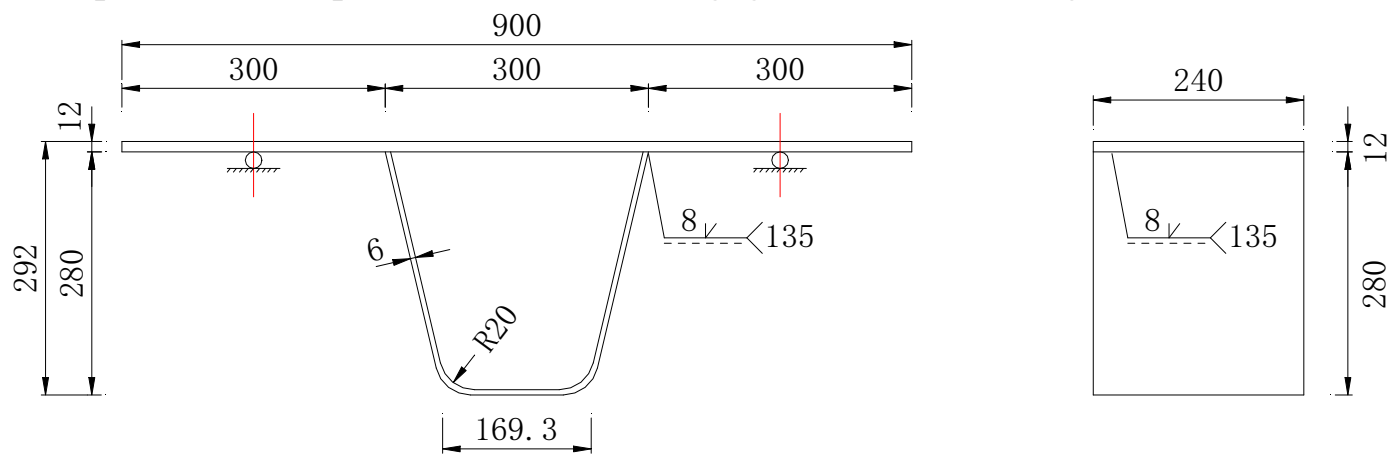

Fig. 1 The size of sectional steel box girder specimen (unit/mm) 


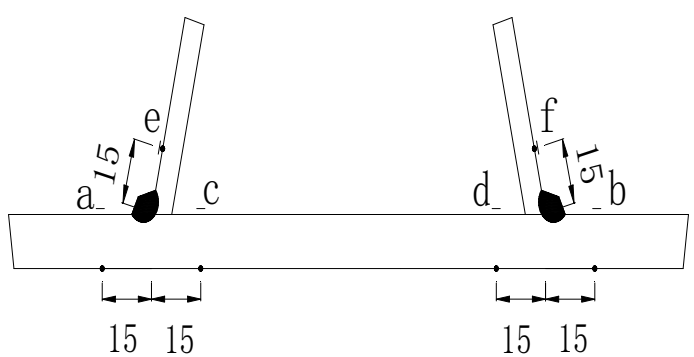

Fig. 2 Layout drawing of strain sensor (unit $/ \mathrm{mm}$ )

\section{Corrosion Experiment Design}

The experiment designs four specimens, which are recorded as JD1 to JD4. The study is the fatigue property of steel box girder in corrosive environment, and the steel box girder of contact corrosion medium is air, therefore, the corrosion of steel box girder is mainly affected by atmospheric corrosion, namely due to the moisture in the air, oxygen, and corrosive medium to cause chemical and electrochemical reactions. The atmospheric corrosion environment divides into rural atmosphere, urban atmosphere, industrial atmosphere, ocean atmosphere, relative to other atmospheric environment, Marine atmosphere usually has highly corrosive, it is better than the ordinary air humidity is big, high salt, high temperature and dry and wet cycle obviously. So this article will select the ocean atmospheric as the corrosion of the corrosion environment, but considering the existing laboratory equipment and other factors, the specific environment controlling equipment called multi functional corrosive sprayer equipment is utilized to imitate the marine atmosphere corrosion.

Designing of the single variable corrosion solution can see the influence of the specimen in corrosion environment intuitively. So we hope to design a concentration of $1 \%, 3 \%$ and $5 \%$ sodium chloride corrosive gas, which is to explore the fatigue damage evolution rule of the specimen in different concentrations of gas corrosion.

\section{Fatigue Experiment Design}

The experiment specimen is specimen 1 to specimen 4 . The loading way is sine wave amplitude loading and the limit is $25 \mathrm{kN} / 5 \mathrm{kN}$. The loading frequency is for $3 \mathrm{~Hz}$.

The specific experiment process is as follows:

Preloading: The ends of the specimen 1 is hinged, single point loading. It used to simulate the uniform effect of wheel load which is placed an area of $300 \mathrm{~mm} \times 200 \mathrm{~mm}$ of a steel plate and a piece of rubber sheet between actuators and the cover plate. The load center is in the center of the cover plate. $10 \mathrm{KN}$ is loading on specimen 3times as preloading before the formal experiment beginning, which purpose is to eliminate loose contact and poor contact between plates, rubber plate and specimen.

Static load for the first time: Load is divided into five load to the fatigue load limit $25 \mathrm{kN}$, namely $0 \mathrm{kN} 、 5 \mathrm{kN} 、 10 \mathrm{kN} 、 15 \mathrm{kN} 、 20 \mathrm{kN}$ and $25 \mathrm{kN}$. The strain gauge reading is record under the load per level after waiting for a period of time, and then unloading to zero.

Dynamic load: After completing the static load test, the fatigue machine is sat to dynamic loading, and the fatigue machine load steady load by repeatedly adjust. When the load cycles $\mathrm{N}$ reached 10000 times, 20000 times, 40000 times, 80000 times, 120000, 160000, 220000, 280000, 340000, 420000,.., stopping first, and then loading the five load to the fatigue load limit $25 \mathrm{kN}$, recording strain gauge reading under each levels, and then unloading to zero ,the last adding dynamic load.

End of the test: The experiment is stopped when testing machine position is overrun, and the number of cyclic loading and fatigue failure characteristics is recorded.

\section{Conclusions}

According to available results of the engineering investigation, the fatigue cracks are inevitably existed in steel bridges because of the environmental pollution, overloading vehicles and larger 
numbers of automobiles. The static and fatigue tests of the orthotropic steel bridge deck models were carried out in this research with the fatigue damage developing laws. An experiment design of corrosive and fatigue property of steel box girder with the specific environment controlling equipment is herein taken into account. Through the data from the testing design, the important characteristic width and length of the fatigue crack can be achieved, which can be used for the grasping of the mechanism of the steel box girder in the future.

\section{Acknowledgments}

This project was finally supported by the National Natural Science Foundation of China (Nos. 11232007 and 11272147), Youth Science and Technology Innovation Fund of NUAA(NS2014003).

\section{References}

[1] J. Zhang, J.S. Ye and C.Q. Wang, Chinese J. Comp. Mech., Vol. 25 (2008), p. 574.

[2] J. Zhang, W.G. Lan and B. Yu, Adv. Mater. Res., Vol. 163-164 (2011), p. 1874.

[3] T. Rousakis, ASCE J. Comp. Constr., Vol. 175 (2013), p. 732.

[4] Z.C. He and Z. Yu: J. Traf. Trans. Eng. Vol. 5 (2005), p. 94.

[5] H. Xia, G. D. Roeckb and N. Zhanga: J. Sound Vib., Vol. 26 (2003), p. 103.

[6] T. Ohkami and Y. Ichikawa, Num. Analy. Method Geom., Vol. 15 (1991), p. 609.

[7] M. Sanayei and M.J. Saletnik: J. Struct. Eng. Vol. 122 (1996), p. 563.

[8] J.S. Ye and J. Zhang: J. Seu Univ: Natural Sci. Edit. Vol. 39 (2009), p. 106. 